\title{
Special Issue on Approximation and Online Algorithms
}

\author{
Thomas Erlebach • Giuseppe Persiano
}

Published online: 21 October 2014

(C) Springer Science+Business Media New York 2014

Approximation and online algorithms deal with problems that are difficult to solve due to two different reasons: Either the search space is too large and complex to efficiently find an optimum solution, or the search space is not completely known as the input for the problem is gradually disclosed during the execution of the algorithm. There is extensive research on approximation and online algorithms, and advances made in one of these fields often contribute to new discoveries in the other.

This special issue of Theory of Computing Systems is devoted to selected papers from the Tenth International Workshop on Approximation and Online Algorithms (WAOA 2012), which took place in Ljubljana, Slovenia, on 13-14 September 2012. Of the 60 papers submitted to WAOA 2012, 22 were accepted for presentation at the workshop, and revised and expanded versions of six papers appear in this special issue. All the contributions presented here were invited to this special issue, but went through the standard refereeing process of Theory of Computing Systems.

The diverse topics of the six papers in this special issue reflect the wide range of problem areas in which the techniques from approximation and online algorithms are crucial.

In their contribution Online Results for Black and White Bin Packing, Balogh et al. introduce an interesting new variant of the bin-packing problem in which items are of two types that must alternate in each bin. They present a 3-competitive algorithm and show that the problem is harder than standard bin packing.

\footnotetext{
T. Erlebach $(\bowtie)$

Department of Computer Science, University of Leicester,

University Road, Leicester, LE1 7RH, UK

e-mail: t.erlebach@leicester.ac.uk

G. Persiano

Dipartimento di Informatica, Università di Salerno,

Fisciano, SA, Italy

e-mail: giuper@dia.unisa.it
} 
In their article Some Anomalies of Farsighted Strategic Behavior, Bilò et al. obtain new bounds on the sequential price of anarchy for several well-known congestion games. Their results are surprising in that they show that farsighted behavior may lead to worse performance than a myopic one.

In Approximating Spanning Trees with Few Branches, Chimani and Spoerhase prove that the problem of maximizing the number of nodes with degree at most two in a spanning tree is APX-hard and present a 6/11-approximation algorithm.

In Advice Complexity of Maximum Independent Set in Sparse and Bipartite Graphs, Dobrev et al. obtain results for the maximum independent set problem in the interesting model of online computation with advice where the algorithm is initially provided a small amount of information about the unknown input. Furthermore, they compare the benefits of advice in the known supergraph setting and the unknown graph setting.

In their contribution On Minimum- and Maximum-Weight Minimum Spanning Trees with Neighborhoods, Dorrigiv et al. deal with the Euclidean minimum spanning tree problem in a setting where the input points are imprecise, meaning that their possible locations are specified as disjoint disks in the plane. They provide approximation algorithms for the problem of choosing point locations so as to minimize or maximize the cost of the spanning tree, and they show that both problems are NP-hard.

Finally, in Probabilistic k-Median Clustering in Data Streams, Lammersen et al. consider the $k$-median clustering problem in a setting where the input is a set of probabilistic points. They define coresets for this probabilistic setting and present algorithms to construct them, showing also how to maintain the Euclidean coreset in data streams.

We would like to thank the authors for contributing to this special issue and the referees for their diligent work in the review process. 